\title{
Neural Correlates of Math Gains Vary Depending on Parental Socioeconomic Status (SES)
}

\author{
Özlem Ece Demir-Lira ${ }^{1,2 *}$, Jérôme Prado ${ }^{3}$ and James R. Booth ${ }^{1,4}$ \\ ' Department of Communication Sciences and Disorders, Northwestern University, Evanston, IL, USA, ${ }^{2}$ Department of \\ Psychology, University of Chicago, Chicago, IL, USA, ${ }^{3}$ Institut des Sciences Cognitives Marc Jeannerod, UMR 5304, Centre \\ National de la Recherche Scientifique - Université de Lyon, Bron, France, ${ }^{4}$ Department of Communication Sciences and \\ Disorders, The University of Texas at Austin, Austin, TX, USA
}

OPEN ACCESS

Edited by: Bert De Smedt, Katholieke Universiteit Leuven, Belgium

Reviewed by:

Gavin Price,

The University of Western Ontario,

Canada

Tanya Marie Evans,

Stanford University, USA

${ }^{*}$ Correspondence:

Özlem Ece Demir-Lira

ece@uchicago.edu

Specialty section:

This article was submitted to

Cognition,

a section of the journal

Frontiers in Psychology

Received: 12 February 2016

Accepted: 30 May 2016

Published: 17 June 2016

Citation:

Demir-Lira ÖE, Prado J and Booth JR (2016) Neural Correlates of Math Gains Vary Depending on

Parental Socioeconomic Status

(SES). Front. Psychol. 7:892. doi: 10.3389/fpsyg.2016.00892
We used functional magnetic resonance imaging (fMRI) to examine the neural predictors of math development, and asked whether these predictors vary as a function of parental socioeconomic status (SES) in children ranging in age from 8 to 13 years. We independently localized brain regions subserving verbal versus spatial processing in order to characterize relations between activation in these regions during an arithmetic task and long-term change in math skill (up to 3 years). Neural predictors of math gains encompassed brain regions subserving both verbal and spatial processing, but the relation between relative reliance on these regions and math skill growth varied depending on parental SES. Activity in an area of the left inferior frontal gyrus (IFG) identified by the verbal localizer was related to greater growth in math skill at the higher end of the SES continuum, but lesser improvements at the lower end. Activity in an area of the right superior parietal cortex identified by the spatial localizer was related to greater growth in math skill at the lower end of the SES continuum, but lesser improvements at the higher end. Results highlight early neural mechanisms as possible neuromarkers of long-term arithmetic learning and suggest that neural predictors of math gains vary with parental SES.

Keywords: socioeconomic status, arithmetic, subtraction, fMRI, longitudinal, children

\section{INTRODUCTION}

Children from disadvantaged backgrounds as a group fall behind their peers in math achievement and math skill growth starting from the early grades (Pungello et al., 1996; Cheadle, 2008; National Center for Education Statistics, 2011). However, some of the children from disadvantaged backgrounds exhibit developmental trajectories that are similar to their peers from more advantaged backgrounds. Whether these children recruit the same neural systems as their peers or recruit alternative systems is not known. In the present study, we used functional magnetic resonance imaging (fMRI) to examine the neural predictors of long-term change in children's math skill, and asked whether these predictors vary as a function of parental socioeconomic status (SES). Identifying early predictors of math skill growth in children from varying backgrounds might aid our understanding of the reasons behind individual differences in math skill growth. Increased understanding of the mechanisms behind these individual differences in turn might have implications for decreasing the achievement gap. 
Mathematics is built upon earlier developing, existing verbal and spatial skills (Dehaene et al., 1999). In solving arithmetical problems, adults and children rely upon a wide network of brain regions, including regions that underlie verbal representations and processing, such as left lateral temporal cortex and inferior frontal cortex, and upon brain regions that underlie spatial representations and processing, such as right intra-parietal sulcus (IPS), precuneus, and posterior superior parietal cortex (Lee, 2000; Dehaene et al., 2003; Schmithorst and Brown, 2004; Andres et al., 2011; Prado et al., 2011; Menon, 2013). In the context of arithmetic processing, activation in verbal networks have been linked to retrieval of arithmetic facts and executive control (Prado et al., 2011), whereas activation in spatial networks have been linked to modality independent representations and procedural manipulation of numerical magnitude (De Smedt et al., 2011).

Although studies have found these regions to be engaged in most participants, activity within this network may vary as a function of task and children's concurrent math skill (Zago et al., 2001; Grabner et al., 2007; Rosenberg-Lee et al., 2009; Cho et al., 2011; De Smedt et al., 2011). For example, De Smedt et al. (2011) showed that 10- to 12-year-old children with lower math skill activated the right intraparietal sulcus to a greater extent than children with higher skill for small addition and subtraction problems. The results were interpreted to suggest that low skill children might use procedural strategies (and rely on spatial neural representations), whereas higher skill children might retrieve arithmetical information from memory (and rely on verbal neural representations).

How the neural differences relate to growth in arithmetic skills is unclear. Some studies have shown that structural and intrinsic functional connectivity predicts math gains (Evans et al., 2015; Jolles et al., 2015). For example, a recent study reported that short-term arithmetic skill gains (8 weeks) after an intervention could be predicted by (1) gray matter volume in the hippocampus and (2) functional connectivity between hippocampus and dorsolateral and ventrolateral prefrontal cortices (as well as basal ganglia), highlighting the role of the hippocampus and memory in the development of arithmetic skills (Supekar et al., 2013). In a longitudinal study, improvement in arithmetic retrieval fluency over a 1-year period was related to hippocampus-neocortical connectivity (Qin et al., 2014). Here, for the first time, we examine the task-based functional neural predictors of long-term change in math skill, i.e., up to 3 years. Importantly, our main focus is on if the neural predictors of math skill growth vary along the SES gradient.

SES-related differences in mathematics are larger on verbal aspects of mathematics, such as verbally presented number combinations, than on spatial aspects, such as non-verbal calculations with disks (Jordan and Levine, 2009). SES-related differences in children's verbal skills are well described and appear to be more robust than differences in spatial skills in other domains as well (Hart and Risley, 1995; Noble et al., 2007). In a recent neuroimaging study, we showed that the neural underpinnings of arithmetic processing vary as a function of SES and children's concurrent math skill level (Demir et al., 2015). Reliance on brain regions that support verbal representations (i.e., middle temporal gyrus, MTG) was related to concurrent math skill to a greater extent for higher than lower SES children. On the contrary, reliance on brain regions that support spatial representations (i.e., IPS) were related to concurrent math skill to a greater extent for lower than higher SES children. Importantly, these differences were observed in a sample where a normative range of parental SES was represented. These results suggest that depending on their parental SES, children might develop adaptations and recruit alternative neural networks to perform at par with their peers.

This previous study left open the question of whether the neural networks that predict growth prospectively vary as function of SES and whether these are the same networks that are concurrently predictive of skill? In the present study, we asked how children's early reliance on verbal and spatial neural systems during elementary arithmetic predicts math skill change, and importantly whether the neural systems that predict change vary as a function of SES. To address these questions, we measured brain activity of 8 - to 13 -year-old children during a single-digit subtraction task, as well as during verbal and spatial localizer tasks. We administered a standardized behavioral measure of math skill before scanning and up to 3 years later (Woodcock et al., 2001). We measured parental SES with parental education and occupation information. We specifically tested if in line with our previous findings, reliance on verbal neural systems would predict math skill growth for higher SES children, whereas reliance on spatial neural systems would predict math skill growth for lower SES children.

We used functional localizer tasks to identify the reliance on brain systems underlying verbal and spatial mechanisms during subtraction. We used a word rhyming task as our verbal localizer and a non-symbolic, dot comparison task as our spatial localizer. Previous literature showed that this word rhyming task taps into verbal representations and successfully localizes verbal neural systems in left temporo-parietal and inferior frontal cortices (Booth, 2010; Prado et al., 2011, 2014). Previous literature showed that this dot comparison task taps into spatio-numerical representations and successfully localizes regions in right intraparietal sulcus, superior parietal lobule and precuneus (Prado et al., 2011, 2014). Importantly, performance on tasks similar to our verbal and spatial localizer tasks relate to mathematical skill, suggesting an overlap between the neural basis of our localizers and mathematical performance (Siegel and Linder, 1984; Hecht et al., 2001; Halberda et al., 2008; Simmons et al., 2008; Krajewski and Schneider, 2009; Piazza et al., 2010).

\section{MATERIALS AND METHODS}

\section{Participants}

Forty-one children were recruited from schools in the greater Chicago area to participate in the $s t u d y^{1}$. All children (1) were native English speakers, (2) were free of past or present neurological or psychiatric disorders, (3) had no history of

\footnotetext{
${ }^{1}$ Fifteen of the children included in the current study overlapped with the previous Demir et al. (2015) study examining concurrent relations between math skill and parental SES and neural basis of arithmetic performance.
} 
reading, oral language, or attention deficits, and (4) scored higher than 80 standard score on full scale IQ as measured by Wechsler Abbreviated Scale of Intelligence (WASI; Weschler, 1999). Data from eight participants were excluded because of excessive movement in the scanner (see criteria below, $n=6$ ), low behavioral accuracy in the scanner (i.e., lower than $40 \%$ in the arithmetic and localizer tasks) and/or response bias in the scanner (i.e., false alarm to misses ratio greater than 2 and false alarm rate greater than $50 \%, n=2$ ). The remaining 33 participants (20 females) were included in the analyses. At the beginning of the study (T1) children were from 8 to 13 years of age (mean age $=10.9, S D=1.5$, range $=8-13.8$ ). At the second visit (T2), children were from 11 to 16 years of age (mean age $=13.4, S D=1.5$, range $=10.6-16.1)$. Written consent was obtained from the children and their parents/guardians. All experimental procedures were approved by the Institutional Review Board at Northwestern University.

\section{Standardized Measures}

Children were administered standardized measures to assess their intellectual and mathematical abilities on entering the study (T1) and after a follow-up period of 2.5 years $(S D=0.16$, range $=2.2-$ 2.8) (T2). We measured IQ by the Verbal (Vocabulary, Similarities) and Performance (Block Design, Matrix Reasoning) subtests of the WASI (Weschler, 1999). Mathematical skill was assessed by the Math Fluency subtest of the Woodcock-Johnson III Tests of Achievement (WJ-III, Woodcock et al., 2001). The Math Fluency subtest requires children to solve as many simple addition, subtraction, and multiplication problems as possible within a 3-min period. The difference in raw score between T1 and T2 was 21 points $(S D=14.2)$. Table 1 summarizes children's performance on standardized tests at T1 and T2.

\section{Socioeconomic Status}

Parental SES information was collected on entering the study (T1). A widely-used measure of SES, the Hollingshead Index, based on primary caregiver education and occupation was used as our measure of child SES (Hollingshead, 1975; Adams and Weakliem, 2011). The education level of the primary caregivers was measured categorically with values ranging between 1 (less than 7 th grade) to 7 (graduate degree). The

TABLE 1 | Means and SDs for behavioral measures.

\begin{tabular}{lllllll}
\hline & \multicolumn{2}{c}{ T1 } & & & \multicolumn{2}{c}{ T2 } \\
\cline { 2 - 3 } \cline { 5 - 7 } & Mean & SD & & Mean & SD \\
\hline IQ (Standardized) & 118.1 & 13.8 & & 120.7 & 14.1 \\
Math fluency (Standardized) & 97.1 & 15.0 & & 94.1 & 13.9 \\
Math fluency (Raw) & 65.8 & & 24.7 & & 85.7 & \\
Subtraction accuracy & $83.3 \%$ & & $17.3 \%$ & & - & \\
Subtraction RT & 1172 & & 295 & & - & - \\
Verbal localizer accuracy & $85.5 \%$ & $11.0 \%$ & & - & - \\
Verbal localizer RT & 1280 & 214 & & - & - \\
Spatial localizer accuracy & $87.6 \%$ & $11.9 \%$ & & - & - \\
Spatial localizer RT & 1051 & 204 & & - & -
\end{tabular}

average Hollingshead education score for our sample was 6 $(S D=0.8)$, with a range from 4.5 to 7 years, corresponding to a college or associates degree. The occupation level of the primary caregivers was measured categorically with values ranging between 1 (farm laborer, menial service worker, student, housewife) and 9 (higher executive, large business owner, major professional). The average Hollingshead occupation score was $6(S D=2.3)$, with a range from 1 to 9 , corresponding to technician, semi-professional or small business owner. Following Hollingshead, SES was calculated using the formula $($ Occupation $\times 7)+($ Education $\times 4),(M=50.9, S D=14.3)$. For 24 children both mother and father were primary caregivers, whereas for nine children mother was the primary caregiver. For children with dual caregivers, average education and highest occupation level was used. For the remaining, the education and occupation information of the primary caregiver was used. Average primary caregiver education and occupation were highly correlated with each other, $r=0.74, p<0.04$.

\section{Arithmetic Task}

Children were administered a single-digit subtraction task in the scanner on entering the study (T1). In each trial of the subtraction task, children were asked to evaluate whether the answer to a single-digit subtraction problem was true or false (Figure 1A). Twenty-four number pairs were used, covering the full range of single-digit subtraction problems (with the exceptions below). Each pair was repeated twice with a true answer (e.g., $5-3=2$ ) and once with a false answer. Thus, children were presented with 72 problems in the main experiment and 24 problems in the practice session. False answers were created by subtracting 1 from the correct answer (e.g., $5-3=1$ ) or by adding 1 or 2 to the correct answer (e.g., $5-3=4$ ). Problems with 0 or 1 as the second operand (e.g., $5-0$ ), tie problems where the first and second operand are identical (e.g., 5 - 5), problems where the correct answer correspond to the second term (e.g., 6 - 3) and problems where the first operand is smaller than the second (e.g., 3 - 5) were not used.

\section{Localizer Tasks}

Children were administered two localizer tasks in the scanner on entering the study (T1). We used a word rhyming task to localize verbal neural systems. In each trial of the verbal localizer, two words were sequentially presented. Children were asked to evaluate whether the two words rhymed or not (Figure 1B). All words were monosyllabic English words with varying orthographic and phonological similarity (e.g., dime lime, pint - mint, grade - laid, press - list). Similarity was manipulated so that responses could not be based on spelling alone. Fouty-eight word pairs were used in the main experiment (24 similar, 24 not similar) and 48 word pairs were used in the practice session. We used a non-symbolic, dot comparison task to localize brain regions that subserve spatial representations. In each trial of the spatial localizer, two dot arrays were sequentially presented (Figure 1C). Children were asked to decide which of the two dot arrays were composed of a larger number of dots. Arrays of 12, 24, and 36 dots were used with varying single dot sizes and cumulative surface area. Seventy-two pairs of dot arrays 


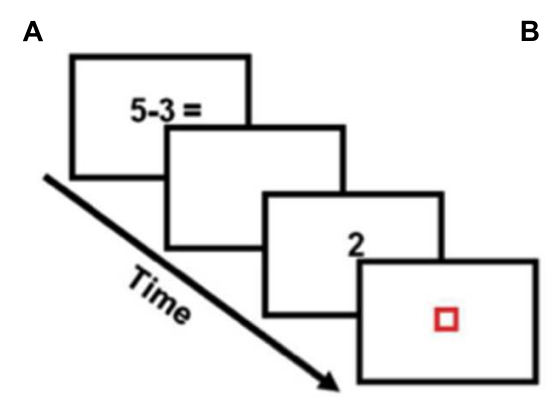

B

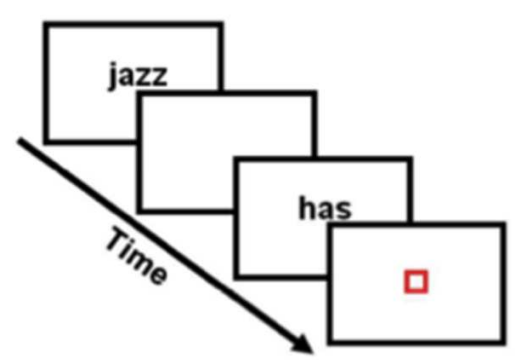

C

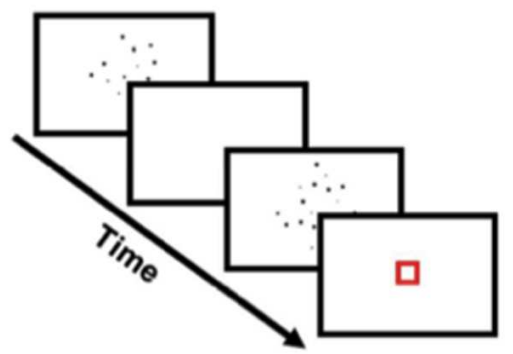

FIGURE 1 | Experimental tasks. (A) In the arithmetic problems, participants were asked to evaluate subtraction problems. Localizer tasks were used to identify the Regions of Interest. (B) In the verbal localizer task, participants decided if two words rhymed or not. (C) In the spatial localizer task, participants decided which dot array included a larger number of dots.

were used in the main experiment and 36 pairs were used in the practice session. Table 1 summarizes children's performance on subtraction and localizer tasks.

\section{Experimental Procedure}

At T1, after informed consent was obtained and standardized tests were administered, children participated in a practice session. During the practice session, children learned to minimize their head movement (with feedback from an infrared tracking device), and practiced all three tasks in a mock fMRI scanner. The actual fMRI scanning session took place within one week of the practice session. In the fMRI scanner, subtraction and spatial localizer tasks were divided into two runs of about $4 \mathrm{~min}$ each. The verbal localizer task was administered in a single run lasting about $7 \mathrm{~min}$. The order of tasks was counterbalanced across participants. Behavioral responses were recorded using an MRcompatible keypad placed below the right hand. Visual stimuli were generated using E-prime software (Psychology Software Tools Inc., 2012), and projected onto a translucent screen. Children viewed the screen through a mirror attached to the head coil.

Stimulus timing was identical in all tasks. A trial started with the presentation of a first stimulus (subtraction, dot array or word depending on the task) for $800 \mathrm{~ms}$, followed by a blank screen for $200 \mathrm{~ms}$. A second stimulus (subtraction, dot array or word depending on the task) was presented for $800 \mathrm{~ms}$, followed by a red fixation square presented for $200 \mathrm{~ms}$. Participants were asked to make a response during an interval ranging from $2,800 \mathrm{~ms}$ to $3,600 \mathrm{~ms}$. Twenty-four null trials were included in the subtraction and spatial localizer tasks. Twelve null trials were used for the verbal localizer task. In the null trials, a blue square was presented for the same duration as the experimental conditions and children were asked to press a button when the square turned red. Each run ended with $22 \mathrm{~s}$ of passive visual fixation. Fixation periods (between trials and at the end of the run) constituted the baseline. The timing and order of trial presentation within each run was optimized for estimation efficiency using Optseq $2^{2}$ (Dale, 1999).

\footnotetext{
${ }^{2}$ http://surfer.nmr.mgh.harvard.edu/optseq/
}

\section{fMRI Data Acquisition}

Images were collected using a Siemens 3T TIM Trio MRI scanner (Siemens Healthcare, Erlangen, Germany) at Northwestern University's Center for Translational Imaging (CTI). The fMRI blood oxygenation level-dependent (BOLD) signal was measured with a susceptibility weighted single-shot echo planar imaging (EPI) sequence. The following parameters were used: $\mathrm{TE}=20 \mathrm{~ms}$, flip angle $=80^{\circ}$, matrix size $=128 \times 120$, field of view $=220 \mathrm{~mm} \times 206.25 \mathrm{~mm}$, slice thickness $=3 \mathrm{~mm}$ (0.48 mm gap), number of slices $=32$, TR $=2,000 \mathrm{~ms}$. Before functional image acquisition, a high resolution T1-weighted 3D structural image was acquired for each subject (TR $=1,570 \mathrm{~ms}$, $\mathrm{TE}=3.36 \mathrm{~ms}$, matrix size $=256 \times 256$, field of view $=240 \mathrm{~mm}$, slice thickness $=1 \mathrm{~mm}$, number of slices $=160$ ).

\section{Behavioral Data Analyses}

The math change score was calculated by subtracting children's raw score on the Math Fluency subtest at T1 from their score at $\mathrm{T} 2$ and dividing this change score by the age difference between $\mathrm{T} 2$ and $\mathrm{T}^{3}$. This measure reflected the rate of change in math score between $\mathrm{T} 1$ and $\mathrm{T} 2$. In order to examine if any of the behavioral measures collected at $\mathrm{T} 1$ predicted math change score, math change score was correlated with IQ, Math Fluency score (raw and standardized score), subtraction accuracy and RT, and age at T1. Correlations with parental SES were also calculated.

\section{fMRI Data Analyses}

Data analyses were performed using SPM8 (Statistical Parametric Mapping ${ }^{4}$. The first six images of each run were discarded, functional images were corrected for slice acquisition delays, realigned to the first image of the first run to correct for head movements, and spatially smoothed with a Gaussian filter equal to about twice the voxel size $(4 \mathrm{~mm} \times 4 \mathrm{~mm} \times 8 \mathrm{~mm}$ full width at half maximum). ArtRepair software was used to suppress residual fluctuations due to large head motion and to identify

\footnotetext{
${ }^{3}$ We used raw scores rather than standardized scores because we were interested in the rate of growth in absolute arithmetic knowledge, rather than relative to peers. Standardized and raw change scores were significantly correlated with each other, $r=0.72, p<0.001$. Results remain unchanged using standardized scores.

${ }^{4}$ www.fil.ion.ucl.ac.uk/spm
} 
volumes with significant artifact and outliers relative to the global mean signal ( $4 \%$ from the global mean). Volumes showing rapid scan-to-scan movements of greater than $1.5 \mathrm{~mm}$ were excluded via interpolation of the two nearest non-repaired volumes. Interpolated volumes were partially deweighted when first-level models were calculated on the repaired images (Mazaika et al., 2009). All participants had less than $5 \%$ of the total number of volumes replaced in a single run. Average translation and rotation movements were small ( $x$-plane: $M=0.06 \mathrm{~mm}$; $y$-plane: $M=0.08 \mathrm{~mm}, z$-plane: $M=0.27 \mathrm{~mm}$, pitch: $M=0.27^{\circ}$, roll: $M=0.12^{\circ}$, yaw: $M=0.09^{\circ}$ ). Functional volumes were coregistered with the segmented anatomical image and normalized to the standard T1 Montreal Neurological Institute (MNI) template volume (normalized voxel size, $2 \mathrm{~mm} \times 2 \mathrm{~mm} \times 4 \mathrm{~mm}$ ).

\section{First Level Analyses}

Event-related statistical analyses were performed according to the General Linear Model. Activation was modeled as epochs with onsets time-locked to the presentation of the first stimulus (operands) and ending at the offset of the second stimulus (answer). For the arithmetic tasks, all responses were included in the model. However, only responses in problems with a true answer were considered of interest in the analyses to avoid inhibitory processes associated with rejecting invalid trials. All epochs were convolved with a canonical hemodynamic response function. The time series data were high-pass filtered $(1 / 128 \mathrm{~Hz})$, and serial correlations were corrected using an autoregressive AR(1) model. Effect sizes were estimated using linear statistical contrasts and subsequently entered into second level analyses.

\section{Second Level Analyses}

In order to evaluate the relations between SES, rate of math score change and neural bases of arithmetic, second level voxelwise regression models were created. In each analysis, SES, math change score, as well as the interaction between SES and math change score constituted the regressors of interest. Additionally, we included as regressor of no interest full scale IQ at T1. Our specific question was about interactive relations of math change score and parental SES to the neural basis of arithmetic. We identified brain regions that showed an increase or a decrease in activity during the evaluation of subtraction problems with respect to the interaction term across subjects. All analyses were repeated with measures of performance (accuracy) on the arithmetic task and T1 math score as regressors of no interest and the results reported below remained unchanged, as described below. We specifically focused on the interaction between math change score and SES because of the nature of our specific question and also in order to reduce our Type 1 error. Analyses examining main effects of math change score and SES on the neural basis of arithmetic are provided in Supplementary Materials.

\section{ROI Definition}

The relations of SES and rate of math change score to the neural basis of subtraction were examined within verbal and spatial ROIs. Verbal ROIs were identified using the verbal localizer contrast (contrast of [words versus null trials] across all subjects). Spatial ROIs were identified using the spatial localizer contrast (contrast of [dots versus null trials]). The resulting statistical maps were thresholded for significance using a voxelwise threshold of $p<0.01$ (uncorrected) and a clusterwise threshold of $p<0.05$ (FWE corrected for multiple comparisons). To ensure the specificity of the localizer activation (i.e., no overlap between localizers), each contrast was exclusively masked by the voxels in which the other localizer contrast was positive (exclusive mask thresholded at $p<0.05$ uncorrected).

The verbal localizer contrast was associated with enhanced activity in the left inferior/middle temporal, inferior/middle frontal, fusiform, and precentral gyri (Figure 2A and Table 2). These clusters constitute the verbal localizer mask. The spatial localizer contrast was associated with enhanced activity in multiple clusters spanning right inferior/superior parietal lobule, precuneus, cuneus, posterior cingulate, lingual gyrus, postcentral gyrus, insula, putamen and left anterior cingulate (Figure $\mathbf{2 B}$ and Table 2). These clusters constituted the spatial localizer mask. The localizers enabled us to independently identify brain regions that subserve verbal versus spatial processes.

\section{ROI Analyses}

Statistical significance within each of these localizer masks was defined using Monte Carlo simulations (using AFNI's AlphaSim program ${ }^{5}$ ). In order to reach corrected level threshold (alpha $=0.05$ ) within the verbal ROIs, the clusters needed to contain 75 voxels with a height threshold of 0.05 . Within the spatial ROIs, clusters needed to contain 85 voxels with a height threshold of 0.05 . Statistical maps were used to estimate smoothness. Throughout the paper, we consider a cluster significant if $p<0.05$ and a trend if $p<0.1$.

\section{Whole Brain Analyses}

To investigate non-predicted effects in regions outside verbal or spatial ROIs, we also report results of whole-brain analyses conducted outside the ROIs reported above. The statistical maps were thresholded for significance using a voxelwise threshold of $p<0.01$ (uncorrected) and a clusterwise threshold of $p<0.05$ (FWE corrected for multiple comparisons).

\section{RESULTS}

\section{Behavioral Performance and Relations to Math Change Score}

Table 3 summarizes correlations between behavioral measures at T1 (e.g., IQ, math score), math change score and SES. None of the behavioral measures significantly predicted change in math scores. We included IQ as a covariate in our analyses, but results remained unchanged using other covariates as described below. SES did not significantly relate to any of the measures at $\mathrm{T} 1$ or to change in math score. In a series of regression analyses, we examined whether math score change was related to the interaction of SES with any of the behavioral measures. We included SES, behavioral measures and their interaction as

\footnotetext{
${ }^{5}$ http://afni.nimh.nih.gov/
} 
A

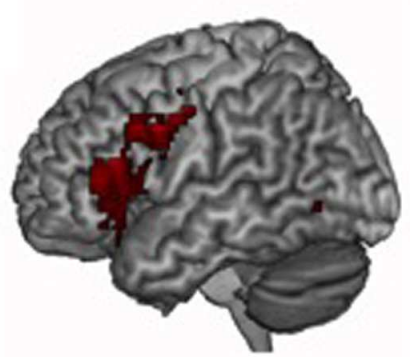

B

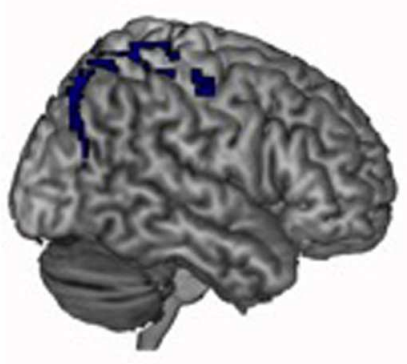

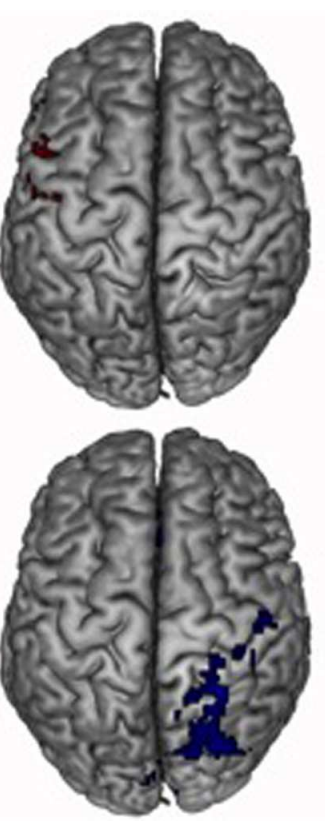
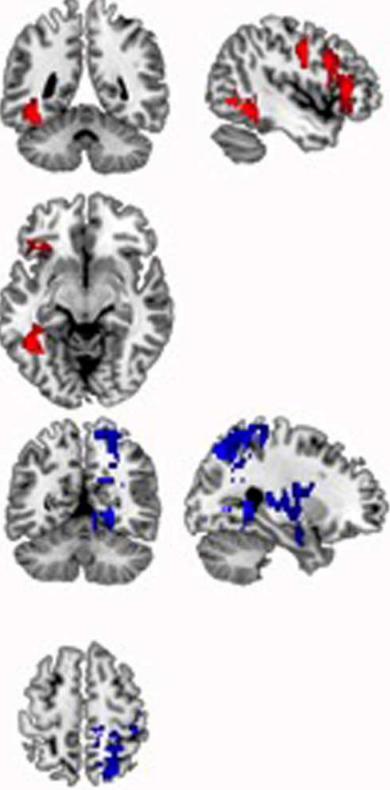

FIGURE 2 | Brain networks identified by the localizer tasks. (A) The verbal localizer task was associated with enhanced activity in a network that included left inferior/middle temporal, inferior/middle frontal, fusiform and precentral gyri. (B) The spatial localizer task was associated with enhanced activity in a network that included right inferior/superior parietal lobule, precuneus, cuneus, posterior cingulate, lingual gyrus, postcentral gyrus, insula, putamen and left anterior cingulate. Activations are overlaid on a 3D rendering and on coronal, sagittal, and axial slices of the MNI-normalized anatomical brain.

independent variables and math change score as the dependent variable. None of the interaction terms predicted math score change.

\section{Overall Activation in Verbal and Spatial ROls during the Subtraction Task}

We first examined overall activation in the verbal and spatial ROIs during the subtraction task, using the contrast of [subtraction trials- baseline] submitted to a one-sample $t$-test across all participants. In verbal ROIs, subtraction problems showed significant activation in left IFG (peak coordinate: $x=-52, y=8, z=38, \mathrm{BA}=9, z=4.21, k=324$ voxels) and in left MTG (peak coordinate: $x=-44, y=-60, z=-6$, $\mathrm{BA}=21, z=3.71, k=178$ voxels). In spatial ROIs, subtraction problems showed activation in right culmen/lingual gyrus (peak coordinate: $x=10, y=-62, z=-14, \mathrm{BA}=19, z=5.52$, $k=109$ voxels), and although not significant, subtraction problems also showed activation in precuneus (peak coordinate: $x=26, y=-46, z=-46, \mathrm{BA}=7, z=3.12, k=51$ voxels).

\section{Relation between Change in Math Score and Neural Activity during the Subtraction Task is Moderated by Parental SES}

We then examined whether SES moderates the relation of rate of math score change to the neural basis of subtraction problems. We identified the brain regions within our verbal or spatial ROIs where activity during the evaluation subtraction problems was

TABLE 2 | Peak activated voxels in the localizer tasks.

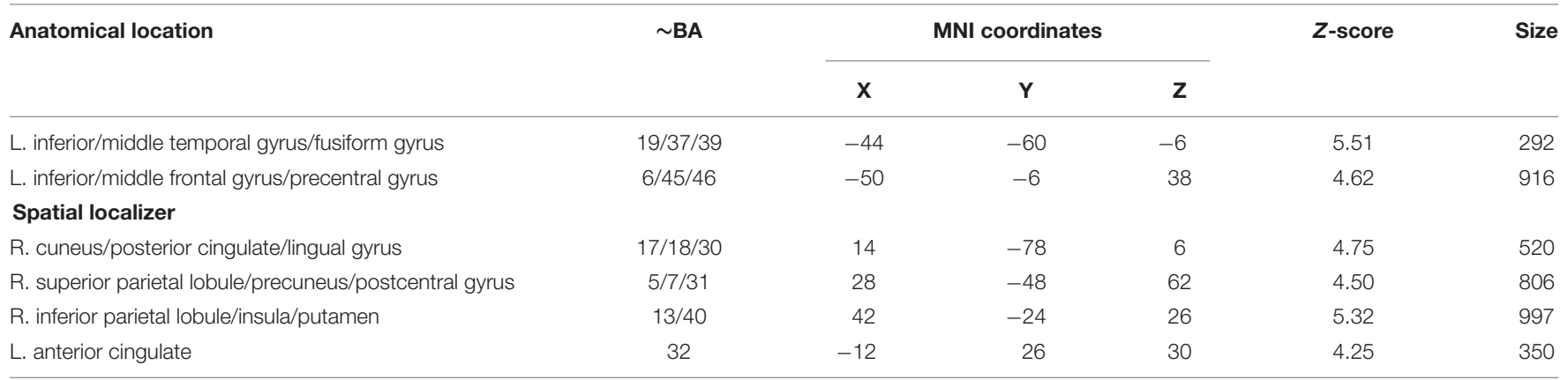

L, left; R, right; $\sim$ BA, approximate Brodmann area for the peak coordinate; MNI, Montreal Neurological Institute; Size, number of $2 \mathrm{~mm} \times 2 \mathrm{~mm} \times 4 \mathrm{~mm}$ voxels. 
TABLE 3 | Correlations between SES, behavioral measures at T1, and change in math fluency.

\begin{tabular}{lcc}
\hline T1 & Change in math fluency & SES \\
\hline SES & 0.01 & - \\
Change in math fluency & - & 0.01 \\
Age & -0.18 & -0.23 \\
WASI IQ (Standardized) & 0.22 & 0.13 \\
WJ math fluency (Standardized) & -0.06 & 0.10 \\
WJ math fluency (Raw) & -0.24 & 0.24 \\
Subtraction accuracy & -0.06 & 0.09 \\
Subtraction RT & -0.09 & 0.23 \\
Verbal localizer accuracy & 0.12 & 0.24 \\
Verbal localizer RT & -0.09 & 0.11 \\
Spatial localizer accuracy & 0.09 & -0.10 \\
Spatial localizer RT & 0.08 & -0.06 \\
\hline
\end{tabular}

None of these correlations were significant.

associated with the interaction between SES and math change score (when the effects of IQ were controlled).

\section{Verbal ROIs}

We found a significant interaction (SES $\times$ change) in a cluster in left IFG (peak coordinate: $x=-48, y=8, z=34$, BA $=9$, $z=3.18, k=79$ voxels; Figure $3 \mathbf{A})^{6}$. For visualization purposes only, we divided the children into two groups based on median SES (lower than or at the median constituting lower SES, and higher than the median constituting higher SES). We then extracted the adjusted eigen variate from the significant cluster and plotted it against math change score for the two SES groups. This plot showed that for higher SES, change is positively associated with activity during subtraction in left IFG, but the relation is negative for lower SES (Figure 3B).

Finally, the interaction identified with continuous variables was confirmed with follow-up analyses comparing relations between change score and activation for higher versus lower SES children. For these analyses, we divided the children into two groups based on median SES (lower than or at the median constituting lower SES, and higher than the median constituting higher SES). We conducted a full factorial design including SES as a binary variable (higher, lower), change as continuous variable, as well as an interaction term between the binary SES variable and change. IQ was included as a continuous covariate. The interaction term enabled us to directly compare the association between change and brain activity in higher versus lower SES. We first identified areas in verbal ROIs where brain activity was associated with change to a greater extent for higher than lower SES children. This direct comparison revealed that a cluster in left IFG was significantly and more strongly related to change in higher SES children than lower SES children (peak coordinate: $x=-50, y=8, z=34, \mathrm{BA}=9, z=3.56, k=187$ voxels). This cluster overlaps with the cluster identified by the analyses using the continuous variables. The reverse contrast did not reveal any

\footnotetext{
${ }^{6}$ The pattern of results for the interaction term in the verbal ROI remained unchanged when controlling for accuracy on task $(z=3.23, k=82)$ and math score at $\mathrm{T} 1(z=3.32, k=71)$.
}

significant activation - there were no significant clusters in verbal ROIs where activation was related to change more strongly for lower than higher SES children.

\section{Spatial ROls}

We found a marginally significant interaction in a cluster in right PSPL/Pr, (peak coordinate: $x=20, y=-66, z=54$, BA $=7$, $z=2.52, p=0.06, k=83$ voxels; Figure $4 \mathrm{~A})^{7}$. For visualization purposes only, we divided the children into two groups based on median SES. We then extracted the adjusted eigenvariate from the significant cluster and plotted it against math change score for the two SES groups. This plot showed that for lower SES children, change is positively associated with activity during subtraction in right PSPL/Pr, but the relation between change and activation in this area is negative for higher SES children (Figure 4B).

In order to confirm the interaction identified with continuous variables, we then divided the children into two groups based on median SES (lower than or at the median constituting lower SES, and higher than the median constituting higher SES). We conducted a full factorial design including SES as a binary variable (higher, lower), change as continuous variable, as well as an interaction term between the binary SES variable and change. IQ was included as a continuous covariate. The interaction term enabled us to directly compare the association between change and brain activity in higher versus lower SES. We identified areas in spatial ROIs where brain activity was associated with change to a greater extent for lower than higher SES children in spatial ROIs. This direct comparison revealed that a cluster in right PSPL/Pr was significantly and more strongly related to change in lower SES children than higher SES children (peak coordinate: $x=20, y=-66, z=54, \mathrm{BA}=7, z=2.96, k=95$ voxels $)$. This cluster overlaps with the cluster identified by the analyses using the continuous variables. The reverse contrast also revealed a significant activation in the spatial ROIs- a cluster in inferior parietal, specifically extending from postcentral gyrus to insula where activation was related to change more strongly for higher SES than lower SES children (peak coordinate in the insula: $x=30, y=-30, z=18, \mathrm{BA}=13, z=2.70, k=250$ voxels).

\section{Whole Brain Analyses}

Outside the ROIs, the interaction term (SES $\times$ change) was significantly and positively related to activation in two clusters. Activity in these clusters was related to greater growth in math skill at the higher end of the SES continuum, but lesser improvements at the lower end. The two clusters included one spanning right supramarginal, superior temporal and extending into inferior parietal gyri (peak coordinate, $x=42, y=-44$, $z=22, \mathrm{BA}=13 / 22 / 40, z=3.81, k=438$ voxels) and another in right inferior frontal, middle frontal, and extending into precentral gyri (peak coordinate, $x=46, y=10, z=30$, BA $=6 / 9$, $z=3.58, k=278$ voxels). The latter peak was within $5 \mathrm{~mm}$ of the peak noted above identified within the verbal ROIs in the left hemisphere. There were no areas that were negatively and significantly related to the interaction term outside the ROIs.

\footnotetext{
${ }^{7}$ The pattern on results for the interaction term in the spatial ROI remained unchanged when controlling for accuracy on task $(z=3.23, k=66)$ and math score at $\mathrm{T} 1(z=2.61, k=92)$.
} 


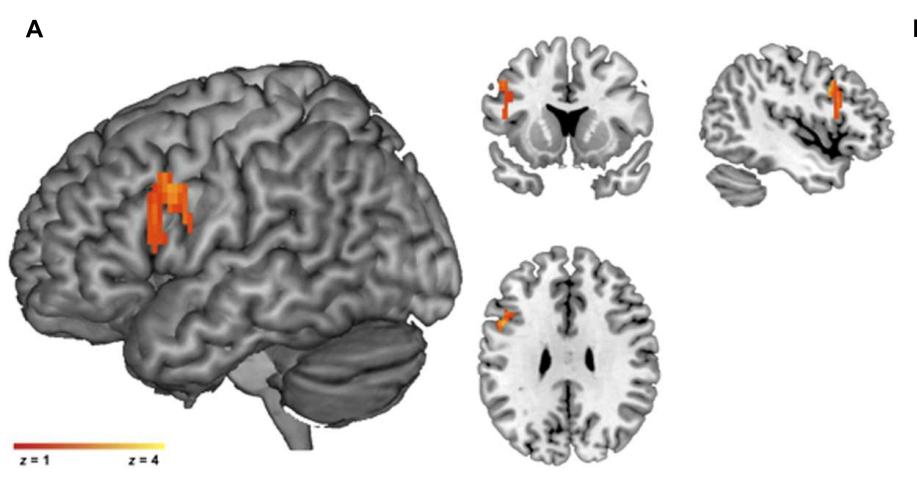

B

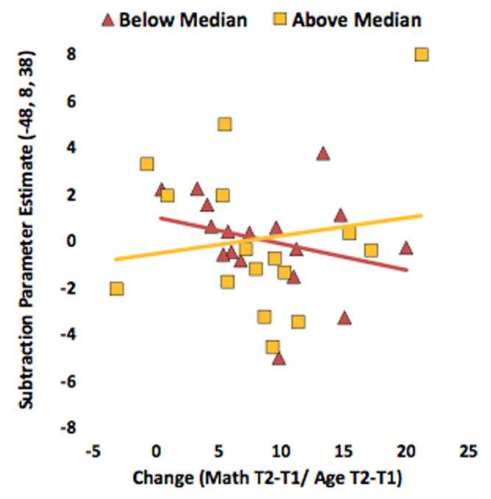

FIGURE 3 | Interaction between SES and math score change in the verbal ROI for subtraction problems. (A) Activity in left inferior frontal gyrus (IFG) showed a SES and change interaction. Activation is overlaid on a 3D rendering and on coronal, sagittal, and axial slices of MNI-normalized anatomical brain. (B) Average brain activity (adjusted eigenvariates) was extracted from the significant cluster in left IFG and plotted against change scores for visualization purposes only. Relation was visualized for children above the median SES and below the median SES.

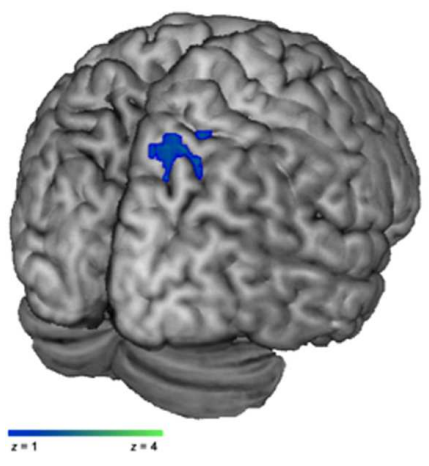

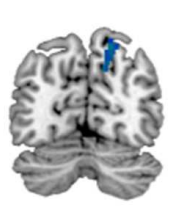

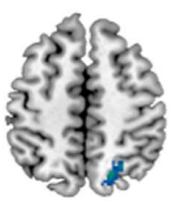

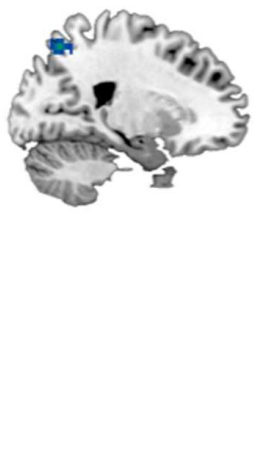

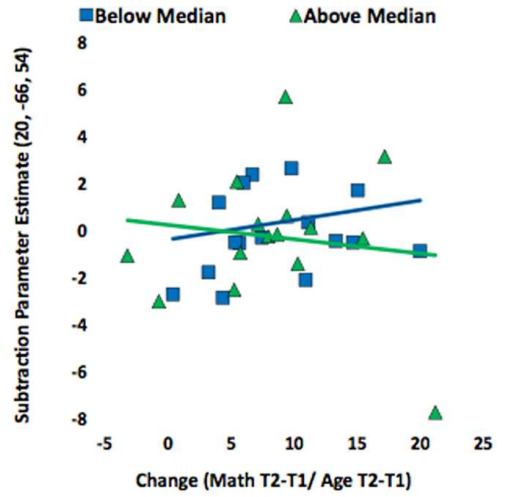

FIGURE 4 | Interaction between SES and math score change in the spatial ROI for subtraction problems. (A) Activity in right superior parietal sulcus/precuneus (PSPL/Pr) showed a SES and change interaction. Activation is overlaid on a 3D rendering and on coronal, sagittal, and axial slices of $\mathrm{MNI}$-normalized anatomical brain. (B) Average brain activity (adjusted eigenvariates) was extracted from the significant cluster (3 mm around the peak) in right PSPL/Pr, and plotted against change scores for visualization purposes only. Relation was visualized for children above the median SES and below the median SES.

\section{DISCUSSION}

Children differ widely in their math skill growth, and parental SES is one of the strongest predictors of these individual differences. To our knowledge, nothing is known about how early neural predictors of later math skill growth vary for children at different SES levels. In the current study, we independently identified brain regions that subserve verbal and spatial neural systems using localizer tasks. We asked how early reliance on these regions relate to growth in math skill over a 3-year period and whether the neural predictors vary as a function of parental SES. Results showed that early neural predictors of math skill gains encompassed brain regions underlying verbal processing, such as left inferior frontal and middle temporal gyri, as well as visuo-spatial processing, such as right culmen/lingual gyrus and precuneus. In addition, neural predictors of math gains varied depending on parental SES.

Activity in an area of the left inferior frontal gyrus (IFG) identified by the verbal localizer was related to greater growth in math skill at the higher end of the SES continuum, but lesser improvements at the lower end. We showed that early reliance on verbal neural systems, specifically left IFG, predicted rate of change in math skill to a greater extent for children at the higher end of the SES continuum than the lower end. Left IFG is consistently activated in arithmetic tasks, and considered to be involved the manipulation of verbal representations of arithmetic rules and facts hosted in left middle temporal cortex (Kucian et al., 2008; Rosenberg-Lee et al., 2009). Left IFG is also implicated in executive control, which is strongly associated with both SES and arithmetic skill (Bull and Scerif, 2001; Badre and Wagner, 2007; Hackman and Farah, 2009). 
During development higher SES children might have learned to better manipulate verbal representations in general and verbal representations of numerical quantities more specifically (for example between Arabic numbers and their meanings) as compared to lower SES children. Differential relations of SES and math skill growth in left IFG might reflect more robust manipulation of such verbal representations by higher SES with higher skill growth children compared to children from lower SES backgrounds. This might aid higher SES children when learning arithmetic problems, e.g., in forming associations between problems and answers or acquiring arithmetic rules and procedures, more than lower SES children. Whole brain analyses revealed right-lateralized activation in right supramarginal/superior temporal and inferior/middle frontal areas to be more strongly associated with math skill change at the higher end of the SES continuum as compared to the lower end. A bilateral fronto-temporal network including these regions has been argued to underlie verbal processing of arithmetic problems, specifically of verbal retrieval or verbalization (Zarnhofer et al., 2012). Thus, whole-brain analyses add support to the interpretation that reliance on verbal representations might predict growth in math skill in higher SES to a greater extent than lower SES children.

In a previous paper, we showed that, at higher levels of SES, higher math skill was associated with concurrent reliance on left MTG, but not IFG (Demir et al., 2015). Left temporoparietal cortices are thought to support verbal representations, such as representations of the associations between arithmetic problems and their solutions (Booth et al., 2002; Fiebach et al., 2002; Blumenfeld et al., 2006; Prado et al., 2011). Combined with the current findings, these results suggest that concurrent math skill might be related to the representational systems themselves hosted in middle temporal regions, whereas acquiring new math knowledge might be associated with 'higher-level' regions manipulating these representations, such inferior frontal regions.

Early parental input might explain why reliance on verbal neural systems predicts growth in math skill to a greater extent for higher than lower SES children. Children differ widely from each other along the SES continuum in their exposure to verbal input in general and verbal input about mathematics specifically, but SES differences in exposure to spatial stimulation are less consistent (Saxe et al., 1987; Hart and Risley, 1995; Blevins-Knabe and Musun-Miller, 1996; Hoff, 2003; Ehrlich, 2007; Levine et al., 2010; Gunderson and Levine, 2011; Levine et al., 2012). The quantity of parental number talk during naturalistic parent-child interactions during preschool years is higher in higher SES families (Gunderson and Levine, 2011). Parental verbal input strongly relates to preschool numerosity outcomes, more strongly than numerosity-related activities (Gunderson and Levine, 2011; Anders et al., 2012). Previous neuroimaging studies suggest that the neural basis of verbal processing, specifically left IFG, is more specialized in higher SES children, confirming our findings regarding left IFG predicting greater change for higher SES children than lower SES children (Pakulak et al., 2005; Raizada et al., 2008; Hackman and Farah, 2009).
Activity in an area of the right superior parietal cortex identified by the spatial localizer was related to greater growth in math skill at the lower end of the SES continuum, but lesser improvements at the higher end. Early reliance on spatial neural systems, specifically right superior parietal cortex/precuneus (PSPL/Pr), predicted rate of change in math skill to a greater extent for lower than higher SES children. The right PSPL/Pr is considered to be involved in spatial and attentional processes and, in the context of arithmetic, in the spatial manipulation of numerical magnitudes, hosted in right intraparietal sulcus (Dehaene et al., 2003; Ischebeck et al., 2006; Metcalfe et al., 2013; Prado et al., 2014; Berteletti et al., 2015). We argue that differential relations of SES and math skill growth in right PSPL/Pr might reflect more robust manipulation of spatial representations of numbers by children with lower SES with higher skill growth. In the absence of the rich verbal input that higher SES children receive, lower SES children might rely on spatial strategies in learning arithmetic to a greater extent than higher SES children. It should be noted that the interaction effect we observed might also be due to higher SES children showing a negative relation to change in right PSPL/Pr - children who use spatial strategies despite being exposed to rich input might exhibit shallower growth over time.

Indeed, SES-related differences in mathematical cognition tend to be larger on verbal aspects of math as compared to spatial aspects (Jordan and Levine, 2009). Interventions aiming to improve mathematical cognition in low SES children are also reported to improve performance on verbal aspects of math, e.g., comparison of number words, but not non-symbolic, spatial aspects, e.g., comparison of magnitudes, suggesting greater room for growth in verbal systems (Wilson et al., 2009). Extending our findings to the domain of reading, Gullick et al. (in press) recently similarly reported that the relation of reading skill to white matter depends on SES. For lower SES children, higher reading skill was correlated with white matter in right hemisphere visuospatial tracts, suggesting that lower SES children may rely more on visuo-spatial orthographic processing strategies for reading success. Thus, lower SES children might find rely on visuo-spatial neural systems to a greater extent than higher SES children across different academic tasks.

Prior literature suggested that SES-related differences in mathematics are larger on verbal aspects of mathematics than on spatial aspects (Jordan and Levine, 2009). In general, SES-related differences in children's verbal skills are well described and appear to be more robust than differences in spatial skills (Hart and Risley, 1995; Noble et al., 2007). Our findings add to the existing literature suggesting that the nature of SES differences might be better described as interacting with children's skill and highlight differential relationships between SES and verbal versus spatial neural systems, rather than an overall effect of SES on verbal systems. In sum, depending on parental SES, children might develop adaptations and recruit alternative neural networks to varying degrees to perform at par with their peers.

In a previous study (Demir et al., 2015), we showed the activation in right IPS to relate to concurrent math skill for children at the lower end of the SES continuum. The IPS has been argued to house spatial representations important for arithmetic 
processing (Dehaene et al., 2003). These results combined with current findings support our argument regarding the distinction between neural systems that support representations themselves for concurrent performance versus manipulation of these representations for learning. Indeed, longitudinal behavioral studies with children showed that working memory is a strong predictor of mathematical skill growth over and above the contributions of domain-specific quantitative, calculation or reading skill, short-term memory and phonological processing skill (Bull et al., 2008; Swanson et al., 2008; Welsh et al., 2010; Metcalfe et al., 2013). Similarly, a recent neuroimaging study found that activation in parietal lobule during a visuo-spatial working memory task predicts math skill growth over a 2-year period (Dumontheil and Klingberg, 2011).

Our results showed that growth in math skill was significantly predicted by neural, but not behavioral measures included in the study, e.g., IQ, early math skill and age. Although null results are hard to interpret, these results are in line recent neuroimaging studies in math and reading development that showed predictive power of neural differences over and above behavioral differences (Hoeft et al., 2007, 2011; McNorgan et al., 2011; Supekar et al., 2013). Neuroimaging measures might serve as sensitive measures of individual differences in underlying neural mechanisms not fully captured by current behavioral standardized tests. This highlights the possibility of using early neural markers to predict future math performance.

The current study raises various questions to be addressed by future research. First, the current study specifically focused on subtraction problems. Prior studies have shown that subtraction problems activate both verbal and visuo-spatial neural systems, and thus subtraction problems might be more appropriate to examine the differential reliance of SES on verbal versus spatial neural systems (Siegler, 1988; De Smedt et al., 2011). However, future studies should examine SES relations to the neural basis of other operations, specifically those that primarily rely on verbal representations, such as multiplication (Lee and Kang, 2002; Prado et al., 2013). Second, our study did not include children at lowest end of the SES continuum. This enabled us to examine SES-related differences within the normative range of SES, in the absence of other confounding factors, such as nutritional differences, differences in sleep patterns or stress. In our study children's behavioral performance on single-digit arithmetic problems did not vary according to SES, which also allowed us to examine SES-related differences without confounding neural effects with differences due to accuracy or motivation. Future studies should examine SESrelated differences in neural predictors of growth in more complex mathematical tasks where SES discrepancies are particularly wide, such as math word problems and on a wider SES continuum (Abedi and Lord, 1998; Jordan and Levine, 2009). Third, SES is a broad measure encompassing multiple characteristics including parental education, occupation, income, perceived social status, and is associated with parental cognitive stimulation, access to education, high-quality neighborhoods, and reduced stress among others (Brooks-Gunn and Duncan, 1997; Bradley and Corwyn, 2002; Hackman and Farah, 2009;
Duncan and Magnuson, 2012). We used widely used indicators of SES that strongly relate to academic outcomes and we controlled for effects of IQ to gain more specificity about SES effects. Future studies should provide further specificity regarding the relations of different components of SES and neural basis of arithmetic development. Finally, it is important highlight that neural predictors of math growth encompassed brain regions that underlie both verbal and spatial processing. It was the relative degree to which activity in an area was related to math gains that varied along the SES continuum. Future longitudinal studies should focus on when do the differences along the SES gradient emerge and develop over time.

In summary, we, for the first time, highlight how neural systems that may be early neural predictors of long-term mathematical learning vary as a function of SES. Reducing the achievement gap necessitates a nuanced understanding of children's differences early on. Although many intervening steps still need to be taken, targeted interventions that build upon early neural indicators might effectively address the challenges of children from differing backgrounds.

\section{AUTHOR CONTRIBUTIONS}

JB directed the larger study in which the present sub-study is embedded. OD-L, JP, and JB substantially contributed to the sub-study conceptualization and design of the work. JB and JP developed the experimental paradigm. OD-L and JP oversaw data collection. OD-L conducted the data processing, data analysis, and interpretation with input from JP and JB. OD-L drafted the manuscript, and all authors provided critical revisions. All authors approved the final version of the manuscript for submission. All authors agree to be accountable for all aspects of the work in ensuring that questions related to the accuracy or integrity of any part of the work are appropriately investigated and resolved.

\section{FUNDING}

This research was supported by HD059177 from the National Institute of Child Health and Human Development to JB.

\section{ACKNOWLEDGMENTS}

We thank John V. Binzak and Rachna Mutreja for their assistance in data collection. We would like to thank all participating children and their parents.

\section{SUPPLEMENTARY MATERIAL}

The Supplementary Material for this article can be found online at: http://journal.frontiersin.org/article/10.3389/fpsyg.2016. 00892 


\section{REFERENCES}

Abedi, J., and Lord, C. (1998). The language factor in mathematics tests. Appl. Meas. Educ. 14, 219-234. doi: 10.1207/S15324818AME1403_2

Adams, J., and Weakliem, D. L. (2011). August B. Hollingshead's "Four factor index of social status": from unpublished paper to citation classic. Yale J. Sociol. 8, $11-20$.

Anders, Y., Rossbach, H.-G., Weinert, S., Ebert, S., Kuger, S., Lehrl, S., et al. (2012). Home and preschool learning environments and their relations to the development of early numeracy skills. Early Child. Res. Q. 27, 231-244. doi: 10.1016/j.ecresq.2011.08.003

Andres, M., Pelgrims, B., Michaux, N., Olivier, E., and Pesenti, M. (2011). Role of distinct parietal areas in arithmetic: an fMRI-guided TMS study. Neuroimage 54, 3048-3056. doi: 10.1016/j.neuroimage.2010.11.009

Badre, D., and Wagner, A. D. (2007). Left ventrolateral prefrontal cortex and the cognitive control of memory. Neuropsychologia 45, 2883-2901. doi: 10.1016/j.neuropsychologia.2007.06.015

Berteletti, I., Man, G., and Booth, J. R. (2015). How number line estimation skills relate to neural activations in single digit subtraction problems. Neuroimage 107, 198-206. doi: 10.1016/j.neuroimage.2014.12.011

Blevins-Knabe, B., and Musun-Miller, L. (1996). Number use at home by children and their parents and its relationship to early mathematical performance. Early Dev. Parent. 5, 35-45. doi: 10.1002/(SICI)1099-0917(199603)5:1<35::AIDEDP113>3.0.CO;2-0

Blumenfeld, H. K., Booth, J. R., and Burman, D. D. (2006). Differential prefrontaltemporal neural correlates of semantic processing in children. Brain Lang. 99, 226-235. doi: 10.1016/j.bandl.2005.07.004

Booth, J. R. (2010). "Development and language," in Encyclopaedia of Behavioral Neuroscience, eds G. Koob, M. Le Moal, and R. F. Thompson (Oxford: Academic Press), 387-395.

Booth, J. R., Burman, D. D., Meyer, J. R., Gitelman, D. R., Parrish, T. B., and Mesulam, M. M. (2002). Modality independence of word comprehension. Hum. Brain Mapp. 16, 251-261. doi: 10.1002/hbm.10054

Bradley, R. H., and Corwyn, R. F. (2002). Socioeconomic development and child development. Annu. Rev. Psychol. 53, 371-399. doi: 10.1146/annurev.psych.53.100901.135233

Brooks-Gunn, J., and Duncan, G. J. (1997). The effects of poverty on children. Future Child. 7, 55-71. doi: 10.2307/1602387

Bull, R., Espy, K. A., and Wiebe, S. A. (2008). Short-term memory, working memory, and executive functioning in preschoolers: longitudinal predictors of mathematical achievement at age 7 years. Dev. Neuropsychol. 33, 205-228. doi: $10.1080 / 87565640801982312$

Bull, R., and Scerif, G. (2001). Executive functioning as a predictor of children's mathematics ability: Inhibition, switching, and working memory. Dev. Neuropsychol. 19, 273-293. doi: 10.1207/S15326942DN1903_3

Cheadle, J. E. (2008). Educational investment, family context, and children's math and reading growth from kindergarten through the third grade. Sociol. Educ. 81, 1-31. doi: 10.1177/003804070808100101

Cho, S., Ryali, S., Geary, D. C., and Menon, V. (2011). How does a child solve 7 +8 ? Decoding brain activity patterns associated with counting and retrieval strategies. Dev. Sci. 14, 989-1001. doi: 10.1111/j.1467-7687.2011.01055.x

Dale, A. M. (1999). Optimal experimental design for event-related fMRI. Hum. Brain Mapp. 8, 109-114.

De Smedt, B., Holloway, I. D., and Ansari, D. (2011). Effects of problem size and arithmetic operation on brain activation during calculation in children with varying levels of arithmetical fluency. Neuroimage 57, 771-781. doi: 10.1016/j.neuroimage.2010.12.037

Dehaene, S., Piazza, M., Pinel, P., and Cohen, L. (2003). Three parietal circuits for number processing. Cogn. Neuropsychol. 20, 487-506. doi: $10.1080 / 02643290244000239$

Dehaene, S., Spelke, E., Pinel, P., Stanescu, R., and Tsivkin, S. (1999) Sources of mathematical thinking: behavioral and brain-imaging evidence. Science 284, 970-974. doi: 10.1126/science.284.5416.970

Demir, ÖE., Prado, J., and Booth, J. R. (2015). Parental socioeconomic status and the neural basis of arithmetic: differential relations to verbal and visuo-spatial representations. Dev. Sci. 18, 799-814. doi: 10.1111/desc.12268
Dumontheil, I., and Klingberg, T. (2011). Brain activity during a visuospatial working memory task predicts arithmetical performance 2 years later. Cereb. Cortex 22, 1078-1085. doi: 10.1093/cercor/bhr175

Duncan, G. J., and Magnuson, K. (2012). Socioeconomic status and cognitive functioning: moving from correlation to causation. Wiley Interdiscip. Rev. Cogn. Sci. 3, 377-386. doi: 10.1002/wcs.1176

Ehrlich, S. (2007). The Preschool Achievement Gap: Are Variations in Teacher Input Associated with Differences in Number Knowledge? Ph.D. dissertation, University of Chicago, Chicago, IL.

Evans, T. M., Kochalka, J., Ngoon, T. J., Wu, S. S., Qin, S., Battista, C., et al. (2015). Brain structural integrity and intrinsic functional connectivity forecast 6 year longitudinal growth in children's numerical abilities. J. Neurosci. 35, 11743-11750. doi: 10.1523/JNEUROSCI.0216-15.2015

Fiebach, C. J., Friederici, A. D., Müller, K., and von Cramon, D. Y. (2002). fMRI evidence for dual routes to the mental lexicon in visual word recognition. J. Cogn. Neurosci. 14, 11-23. doi: 10.1162/089892902317205285

Grabner, R. H., Ansari, D., Reishofer, G., Stern, E., Ebner, F., and Neuper, C. (2007). Individual differences in mathematical competence predict parietal brain activation during mental calculation. Neuroimage 38, 346-356. doi: 10.1016/j.neuroimage.2007.07.041

Gullick, M., Demir, ÖE., and Booth, J. R. (in press). Socio-economic status predicts divergent reading skill-fractional (anisotropy)relationships in visuospatial tracts. Dev. Sci.

Gunderson, E. A., and Levine, S. C. (2011). Some types of parent number talk count more than others: relations between parents' input and children's cardinal-number knowledge. Dev. Sci. 14, 1021-1032. doi: 10.1111/j.14677687.2011.01050.x

Hackman, D. A., and Farah, M. J. (2009). Socioeconomic status and the developing brain. Trends Cogn. Sci. 13, 65-73. doi: 10.1016/j.tics.2008.11.003

Halberda, J., Mazzocco, M. M., and Feigenson, L. (2008). Individual differences in non-verbal number acuity correlate with maths achievement. Nature 455 , 665-668. doi: 10.1038/nature07246

Hart, B., and Risley, T. R. (1995). Meaningful Differences in the Everyday Experience of Young American Children. Baltimore, MD: Paul H Brookes Publishing Co.

Hecht, S. A., Torgesen, J. K., Wagner, R. K., and Rashotte, C. A. (2001). The relations between phonological processing abilities and emerging individual differences in mathematical computation skills: a longitudinal study from second to fifth grades. J. Exp. Child Psychol. 79, 192-227. doi: 10.1006/jecp.2000.2586

Hoeft, F., McCandliss, B. D., Black, J. M., Gantman, A., Zakerani, N., Hulme, C., et al. (2011). Neural systems predicting long-term outcome in dyslexia. Proc. Natl. Acad. Sci. U.S.A. 108, 361-366. doi: 10.1073/pnas.1008950108

Hoeft, F., Ueno, T., Reiss, A. L., Meyler, A., Whitfield-Gabrieli, S., Glover, G. H., et al. (2007). Prediction of children's reading skills using behavioral, functional, and structural neuroimaging measures. Behav. Neurosci. 121, 602-613. doi: 10.1037/0735-7044.121.3.602

Hoff, E. (2003). The specificity of environmental influence: socioeconomic status affects early vocabulary development via maternal speech. Child Dev. 74, 13681378. doi: 10.1111/1467-8624.00612

Hollingshead, A. B. (1975). Four Factor Index of Social Status. New Haven, CT: Yale University.

Ischebeck, A., Zamarian, L., Siedentopf, C., Koppelstätter, F., Benke, T., Felber, S., et al. (2006). How specifically do we learn? Imaging the learning of multiplication and subtraction. Neuroimage 30, 1365-1375.

Jolles, D., Wassermann, D., Chokhani, R., Richardson, J., Tenison, C., Bammer, R., et al. (2015). Plasticity of left perisylvian white-matter tracts is associated with individual differences in math learning. Brain Struct. Funct. 221, 1337-1351. doi: 10.1007/s00429-014-0975-6

Jordan, N. C., and Levine, S. C. (2009). Socioeconomic variation, number competence, and mathematics learning difficulties in young children. Dev. Disabil. Res. Rev. 15, 60-68. doi: 10.1002/ddrr.46

Krajewski, K., and Schneider, W. (2009). Exploring the impact of phonological awareness, visual-spatial working memory, and preschool quantity-number competencies on mathematics achievement in elementary school: findings from a 3-year longitudinal study. J. Exp. Child Psychol. 103, 516-531. doi: 10.1016/j.jecp.2009.03.009 
Kucian, K., von Aster, M., Loenneker, T., Dietrich, T., and Martin, E. (2008). Development of neural networks for exact and approximate calculation: a FMRI study. Dev. Neuropsychol. 33, 447-473. doi: 10.1080/87565640802101474

Lee, K.-M. (2000). Cortical areas differentially involved in multiplication and subtraction: a functional magnetic resonance imaging study and correlation with a case of selective acalculia. Ann. Neurol. 48, 657-661. doi: 10.1002/15318249(200010)48:4<657::AID-ANA13>3.0.CO;2-K

Lee, K.-M., and Kang, S.-Y. (2002). Arithmetic operation and working memory: differential suppression in dual tasks. Cognition 83, B63-B68. doi: 10.1016/S0010-0277(02)00010-0

Levine, S. C., Ratliff, K. R., Huttenlocher, J., and Cannon, J. (2012). Early puzzle play: a predictor of preschoolers' spatial transformation skill. Dev. Psychol. 48, 530-542. doi: 10.1037/a0025913

Levine, S. C., Suriyakham, L. W., Rowe, M. L., Huttenlocher, J., and Gunderson, E. A. (2010). What counts in the development of young children's number knowledge? Dev. Psychol. 46, 1309-1319. doi: 10.1037/a0019671

Mazaika, P., Hoeft, F., Glover, G., and Reiss, A. (2009). "Methods and software for fMRI analysis for clinical subjects," in Proceedings of the Presentation at the 15th Annual Meeting of the Organization for Human Brain Mapping, San Francisco, CA, 18-23.

McNorgan, C., Alvarez, A., Bhullar, A., Gayda, J., and Booth, J. R. (2011). Prediction of reading skill several years later depends on age and brain region: implications for developmental models of reading. J. Neurosci. 31, 9641-9648. doi: 10.1523/JNEUROSCI.0334-11.2011

Menon, V. (2013). "Arithmetic in child and adult brain," in Handbook of Mathematical Cognition, eds R. C. Kadosh and A. Dowker (Oxford: Oxford University Press).

Metcalfe, A. W., Ashkenazi, S., Rosenberg-Lee, M., and Menon, V. (2013). Fractionating the neural correlates of individual working memory components underlying arithmetic problem solving skills in children. Dev. Cogn. Neurosci. 6, 162-175. doi: 10.1016/j.dcn.2013.10.001

National Center for Education Statistics (2011). The Nation's Report Card: Mathematics 2011. Washington, DC: National Center for Education Statistics.

Noble, K. G., McCandliss, B. D., and Farah, M. J. (2007). Socioeconomic gradients predict individual differences in neurocognitive abilities. Dev. Sci. 10, 464-480. doi: 10.1111/j.1467-7687.2007.00600.x

Pakulak, E., Sanders, L., Paulsen, D. J., and Neville, H. (2005). "Semantic and syntactic processing in children from different familial socio-economic status as indexed by ERPS," in Poster Presented at the 12th Annual Cognitive Neuroscience Society Meeting, New York, NY.

Psychology Software Tools Inc. (2012). E-Prime 2.0 [Computer Software]. Available at: http://www.pstnet.com.

Piazza, M., Facoetti, A., Trussardi, A. N., Berteletti, I., Conte, S., Lucangeli, D., et al. (2010). Developmental trajectory of number acuity reveals a severe impairment in developmental dyscalculia. Cognition 116, 33-41. doi: 10.1016/j.cognition.2010.03.012

Prado, J., Lu, J., Liu, L., Dong, Q., Zhou, X., and Booth, J. R. (2013). The neural bases of the multiplication problem-size effect across countries. Front. Hum. Neurosci. 7:189. doi: 10.3389/fnhum.2013.00189

Prado, J., Mutreja, R., and Booth, J. R. (2014). Developmental dissociation in the neural responses to simple multiplication and subtraction problems. Dev. Sci. 17, 537-552. doi: 10.1111/desc. 12140

Prado, J., Mutreja, R., Zhang, H., Mehta, R., Desroches, A. S., Minas, J. E., et al. (2011). Distinct representations of subtraction and multiplication in the neural systems for numerosity and language. Hum. Brain Mapp. 32, 1932-1947. doi: 10.1002/hbm.21159

Pungello, E. P., Kupersmidt, J. B., Burchinal, M. R., and Patterson, C. J. (1996). Environmental risk factors and children's achievement from middle childhood to early adolescence. Dev. Psychol. 32, 755-767. doi: 10.1037/a00 19816

Qin, S., Cho, S., Chen, T., Rosenberg-Lee, M., Geary, D. C., and Menon, V. (2014). Hippocampal-neocortical functional reorganization underlies children's cognitive development. Nat. Neurosci. 17, 1263-1269. doi: 10.1038/nn.3788
Raizada, R. D. S., Richards, T. L., Meltzoff, A., and Kuhl, P. K. (2008). Socioeconomic status predicts hemispheric specialisation of the left inferior frontal gyrus in young children. Neuroimage 40, 1392-1401. doi: 10.1016/j.neuroimage.2008.01.021

Rosenberg-Lee, M., Lovett, M. C., and Anderson, J. R. (2009). Neural correlates of arithmetic calculation strategies. Cogn. Affect. Behav. Neurosci. 9, 270-285. doi: 10.3758/CABN.9.3.270

Saxe, G. B., Guberman, S. R., Gearhart, M., Gelman, R., Massey, M., and Rogoff, B. (1987). Social processes in early number development. Monogr. Soc. Res. Child Dev. 52:i+iii-v+vii-viii+1+3-17+19+21-33+35-53+55+57-99+101-162. doi: $10.2307 / 1166071$

Schmithorst, V. J., and Brown, R. D. (2004). Empirical validation of the triple-code model of numerical processing for complex math operations using functional MRI and group independent component analysis of the mental addition and subtraction of fractions. Neuroimage 22, 1414-1420. doi: 10.1016/j.neuroimage.2004.03.021

Siegel, L. S., and Linder, B. A. (1984). Short-term memory processes in children with reading and arithmetic learning disabilities. Dev. Psychol. 20, 200. doi: 10.1080/13803395.2015.1066759

Siegler, R. S. (1988). Strategy choice procedures and the development of multiplication skill. J. Exp. Psychol. Gen. 117, 258-275. doi: 10.1037/00963445.117.3.258

Simmons, F., Singleton, C., and Horne, J. (2008). Phonological awareness and visual-spatial sketchpad functioning predict early arithmetic attainment: evidence from a longitudinal study. Eur. J. Cogn. Psychol. 20, 711-722. doi: $10.1080 / 09541440701614922$

Supekar, K., Swigart, A. G., Tenison, C., Jolles, D. D., Rosenberg-Lee, M., and Fuchs, L. (2013). Neural predictors of individual differences in response to math tutoring in primary-grade school children. Proc. Natl. Acad. Sci. U.S.A. 110, 8230-8235. doi: 10.1073/pnas.1222154110

Swanson, H. L., Jerman, O., and Zheng, X. (2008). Growth in working memory and mathematical problem solving in children at risk and not at risk for serious math difficulties. J. Educ. Psychol. 100, 343-379. doi: 10.1037/00220663.100.2.343

Welsh, J. A., Nix, R. L., Blair, C., Bierman, K. L., and Nelson, K. E. (2010). The development of cognitive skills and gains in academic school readiness for children from low-income families. J. Educ. Psychol. 102, 43-53. doi: $10.1037 / \mathrm{a} 0016738$

Weschler, D. (1999). Weschler Abbreviated Scale of Intelligence. New York, NY: The Psychological Corporation.

Wilson, A. J., Dehaene, S., Dubois, O., and Fayol, M. (2009). Effects of an adaptive game intervention on accessing number sense in kindergarten children. Mind Brain Educ. 3, 224-234. doi: 10.1111/j.1751-228X.2009.01075.x

Woodcock, R. W., McGrew, K. S., and Mather, N. (2001). Woodcock-Johnson III Tests of Achievement. Itasca: Riverside Publishing.

Zago, L., Pesenti, M., Mellet, E., Crivello, F., Mazoyer, B., and TzourioMazoyer, N. (2001). Neural correlates of simple and complex mental calculation. Neuroimage 13, 314-327. doi: 10.1006/nimg.2000. 0697

Zarnhofer, S., Braunstein, V., Ebner, F., Koschutnig, K., Neuper, C., Reishofer, G., et al. (2012). The influence of verbalization on the pattern of cortical activation during mental arithmetic. Behav. Brain Funct. 8:1. doi: 10.1186/1744-9081-8-13

Conflict of Interest Statement: The authors declare that the research was conducted in the absence of any commercial or financial relationships that could be construed as a potential conflict of interest.

Copyright (c) 2016 Demir-Lira, Prado and Booth. This is an open-access article distributed under the terms of the Creative Commons Attribution License (CC BY). The use, distribution or reproduction in other forums is permitted, provided the original author(s) or licensor are credited and that the original publication in this journal is cited, in accordance with accepted academic practice. No use, distribution or reproduction is permitted which does not comply with these terms. 